\title{
SQUAMOUS CELL CARCINOMA OF THYROGLOSSAL DUCT CYST
}

\author{
Kiran Bhojwani' ${ }^{1}$ Jyothi Kini², Karthik M³ ${ }^{3}$ Neethu Mary Mathew ${ }^{4}$
}

1 Professor \& HOD, Department of ENT, Mangalore Kasturba Medical College, Mangalore Manipal University. 2Professor, Department of Pathology, Mangalore Kasturba Medical College, Mangalore Manipal University. ${ }_{3}^{3}$ Post Graduate, Department of ENT, Mangalore Kasturba Medical College, Mangalore Manipal University. ${ }^{4}$ Post Graduate, Department of ENT, Mangalore Kasturba Medical College, Mangalore Manipal University.

\section{ABSTRACT}

\section{BACKGROUND}

Incomplete atrophy of thyroglossal tract or retained epithelial cysts, creates the basis for the origin of a thyroglossal duct cyst. Thyroglossal duct cysts are the most common anomaly in thyroid development that occurs in $7 \%$ of adult population. In general duct cysts are benign and the incidence of a carcinoma in a thyroglossal duct cyst is very rare, the incidence of which is $1 \%$ of all cysts. The vast majority of these are papillary carcinoma (82\%), other types of malignancies occurring being mixed papillary and follicular carcinoma, squamous cell carcinoma, follicular carcinoma and anaplastic carcinoma in descending order of frequency. Review of literature has revealed 25 reported cases of squamous cell carcinoma of the thyroglossal duct cyst till date.[1]

\section{CASE REPORT}

We present a case of a forty nine year old male patient, who presented with a swelling in front of the neck which gradually appeared over 4 months with associated change in voice. Further investigations revealed a squamous cell carcinoma with features suggesting the site of origin to be the thyroglossal duct. Sistrunk's operation was done under general anaesthesia, followed by postoperative concurrent chemoradiotherapy.

\section{CONCLUSION}

Malignancy, although rare, within a thyroglossal duct cyst should be included in the differential diagnosis of neck masses. Aggressive treatment in the form of a combined modality of surgery, radiation and chemotherapy is usually the preferred plan of treatment.

\section{KEYWORDS}

Thyroglossal Duct Cyst, Squamous Cell Carcinoma, Sistrunk's Operation.

HOW TO CITE THIS ARTICLE: Bhojwani K, Kini J, Karthik M, et al. Squamous cell carcinoma of thyroglossal duct cyst. J. Evolution Med. Dent. Sci. 2016;5(31):1683-1686, DOI: 10.14260/jemds/2016/396

\section{INTRODUCTION}

Incomplete atrophy of thyroglossal tract or retained epithelial cysts, creates the basis for the origin of a thyroglossal duct cyst. [2] Thyroglossal duct cysts are the most common anomaly in thyroid development that occurs in $7 \%$ of adult population. ${ }^{[3]}$ In general duct cysts are benign and the incidence of a carcinoma in a thyroglossal duct cyst is very rare, the incidence of which is $1 \%$ of all cysts. The vast majority of these are papillary carcinoma (82\%), other types of malignancies occurring being mixed papillary and follicular carcinoma, squamous cell carcinoma, follicular carcinoma and anaplastic carcinoma in descending order of frequency.

The presentation of a case of thyroglossal duct cyst carcinoma is similar to that of a benign duct cyst, which may manifest as a midline neck mass. $\left.{ }^{4]}\right]$

\section{Case Presentation}

A 49-year-old male patient had presented to the ENT OPD with a swelling in front of his neck noticed since 4 months and change in voice since 1 month.

Financial or Other, Competing Interest: None.

Submission 21-01-2016, Peer Review 19-02-2016,

Acceptance 27-02-2016, Published 18-04-2016.

Corresponding Author:

Dr. Kiran Bhojwani,

Professor and HOD,

Department of ENT,

Kasturba Medical College Hospital,

Attavar,

Mangalore-575001.

E-mail: 10sunbeams@gmail.com

DOI: $10.14260 /$ jemds $/ 2016 / 396$
The swelling had an insidious onset, was initially small in size and it gradually progressed in size. It was associated with a dull aching pain. Patient also gave a history of smoking since 25 years. There was no history of weight loss nor appetite loss and no history of fever.

On physical examination, the general condition of the patient was fair and his vitals were stable. Local examination of the neck revealed a solitary $3 \times 2 \mathrm{~cm}$ midline anterior neck swelling with a smooth surface (Figure 1 and Figure 2). The swelling was present $3 \mathrm{~cm}$ above the suprasternal notch which was non-tender, smooth surfaced and hard in consistency. The swelling was seen to be moving with deglutition. Videolaryngoscopy was done which revealed congestion and an erythematous patch present over the posterior third of the right vocal cord. Both cords were moving equally with respiration and phonation and no other abnormalities were detected. The rest of the larynx and hypopharynx was normal.

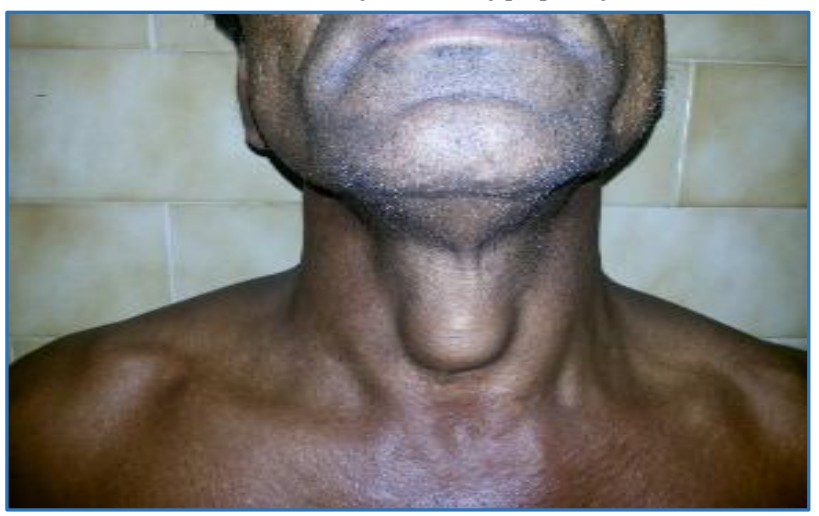

Fig. 1: Anterior view of the Neck Swelling 


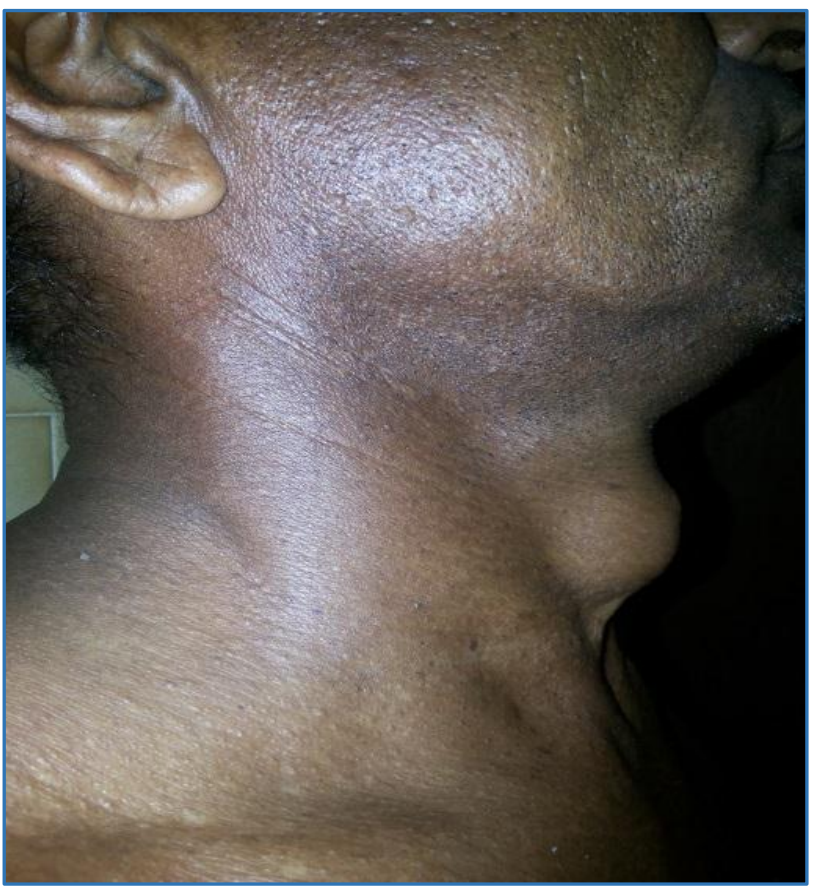

Fig. 2: Lateral view of the Neck Swelling

Routine blood investigations and thyroid function tests were done, which were normal. Computed Tomography (CT) of the neck with contrast showed a well-defined minimally enhancing soft tissue density $1.6 \mathrm{~cm} \times 3.3 \mathrm{~cm}$ in the subcutaneous aspect of the anterior midline of the neck. A few subcentimeter lymph nodes in right level $1 \mathrm{~b}$ and 2 were also noted. There was no evidence of erosion of the thyroid cartilage on CT scan. The thyroid gland was visualized to be normal on CT scan. Fine Needle Aspiration Cytology (FNAC) of the neck swelling was reported as squamous cell carcinoma. Pan-endoscopy was performed under general anaesthesia to rule out any possible foci of primary malignancy. Biopsy from the right vocal cord revealed a moderate dysplasia on histopathological examination. Ultrasound imaging of the abdomen was performed and no anomaly or evidence of malignancy was seen.

As the thyroid was normal on CT scan and no other primaries were detected, a possibility of thyroglossal duct carcinoma was suspected. A Sistrunk's operation was done under general anaesthesia. Intraoperatively, a wellcircumscribed, well-defined midline swelling with a smooth surface and well-defined margins was found. It was firm in consistency and was lying on the thyroid cartilage, overlying the cricothyroid space. The swelling did not involve the perichondrial layer of the thyroid cartilage. In addition, a firm, mobile, single, level $1 \mathrm{~b}$ lymph node of $1 \times 1.5 \mathrm{cms}$ was found. Both lobes and isthmus of the thyroid gland were found to be normal intraoperatively. The thyroglossal duct mass was excised in toto along with the tract going upwards including a part of the hyoid bone, up to the foramen caecum (Figure 3 and Figure 4). The left level $1 \mathrm{~b}$ lymph node was also excised. Both specimens were sent for histopathological evaluation.

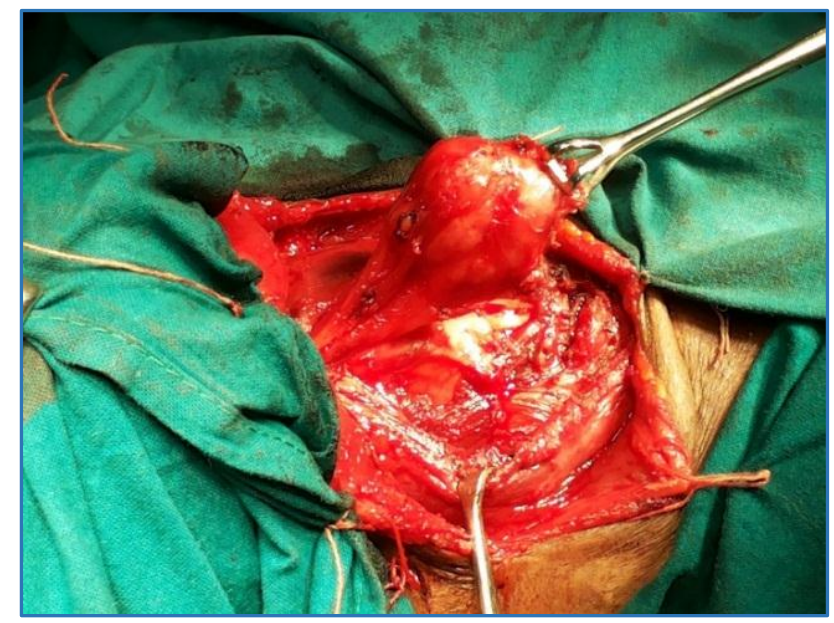

Fig. 3: Intraoperative picture of the Thyroglossal Duct Cyst

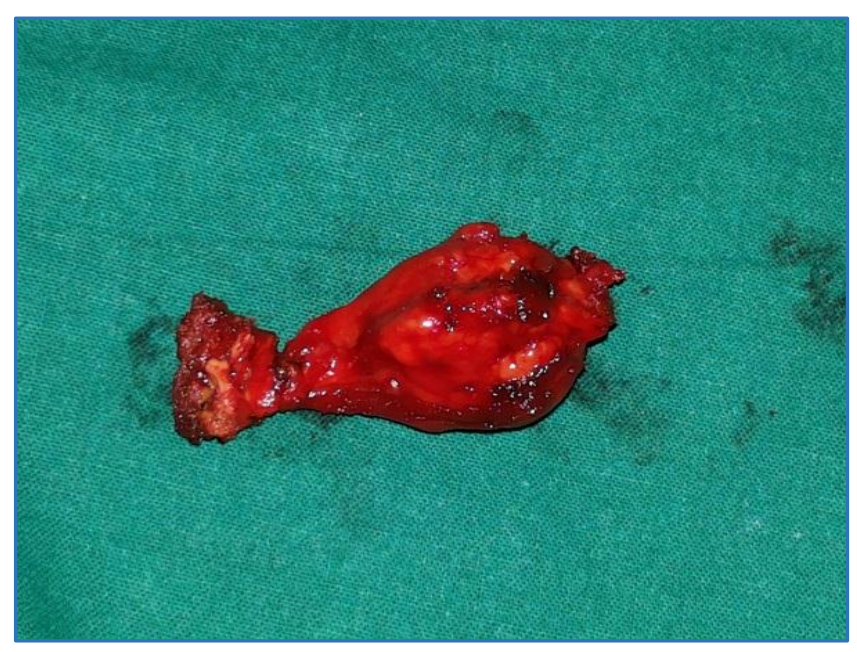

Fig. 4: Thyroglossal Duct Cyst Excised Specimen

On evaluation of the gross appearance of the main specimen, an encapsulated cystic mass with attached muscular tissue on the superior aspect and attached bone fragment was seen. The mass was measuring $2.5 \times 2 \times 2 \mathrm{~cm}$. Cut section through the mass showed encapsulation with a lobulated, finely granular, grey-white, firm, solid tumour. Grossly, the lymph node appeared as a pale brown nodule measuring $1.5 \mathrm{x}$ $1 \times 1 \mathrm{~cm}$ with a pale white appearance on cut section with specks of haemorrhage.

On microscopy, the main specimen of the midline mass was noted to have a thin fibrous capsule with thyroid follicles lined by follicular cells and filled with colloid. The mass contained a tumour composed of atypical malignancy squamous cells in sheets. The cells had a high nuclear to cytoplasmic ratio with coarse granular chromatin, some having prominent nucleoli with moderate amount of eosinophilic cytoplasm. Atypical mitotic figures were also noted. The resected margin was close to the tumour in one focus. Thyroglossal duct cells were also noted alongside the sheets of malignant squamous cells. The excised lymph node showed metastatic deposits of moderately differentiated squamous cell carcinoma. The final report of the excised specimen along with the lymph nodes was moderately differentiated squamous cell carcinoma with resected margins being close to the tumour in one area with lymph node showing positivity for metastasis. 


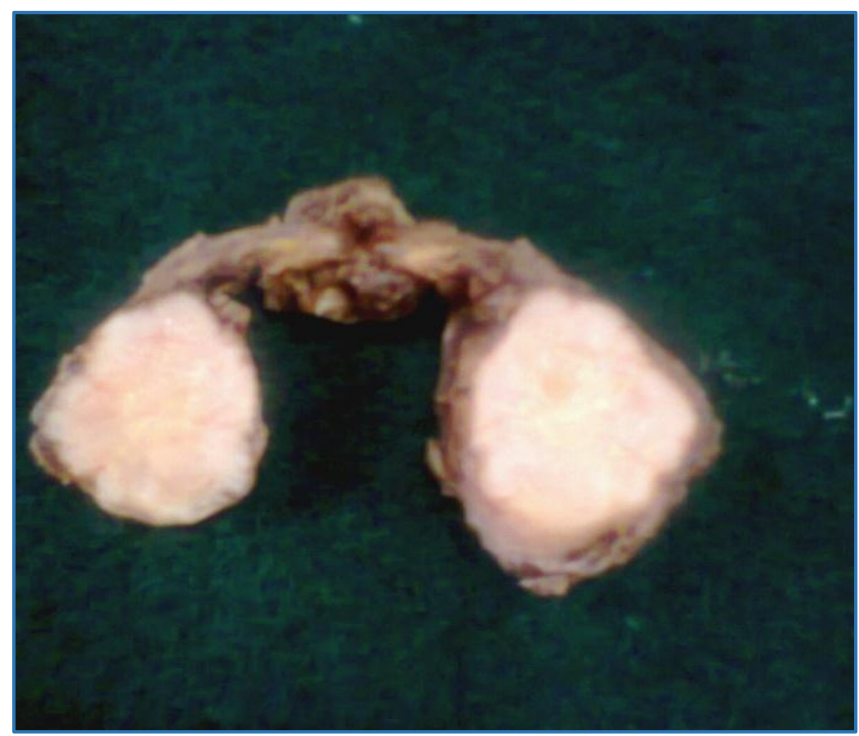

Fig. 5: Gross image of Cut Section of Cyst showing Encapsulated Grey-White Solid, Granular Lesion

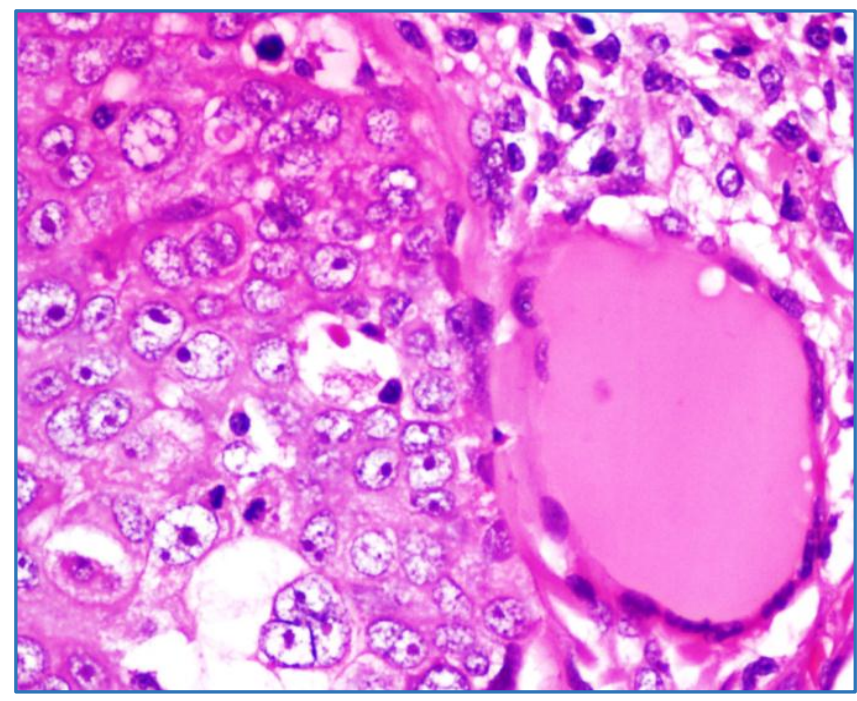

Fig. 6: Cells have high Nuclear:Cytoplasmic ratio with Coarse Granular Chromatin and Prominent Nucleoli with Moderate Eosinophilic Cytoplasm

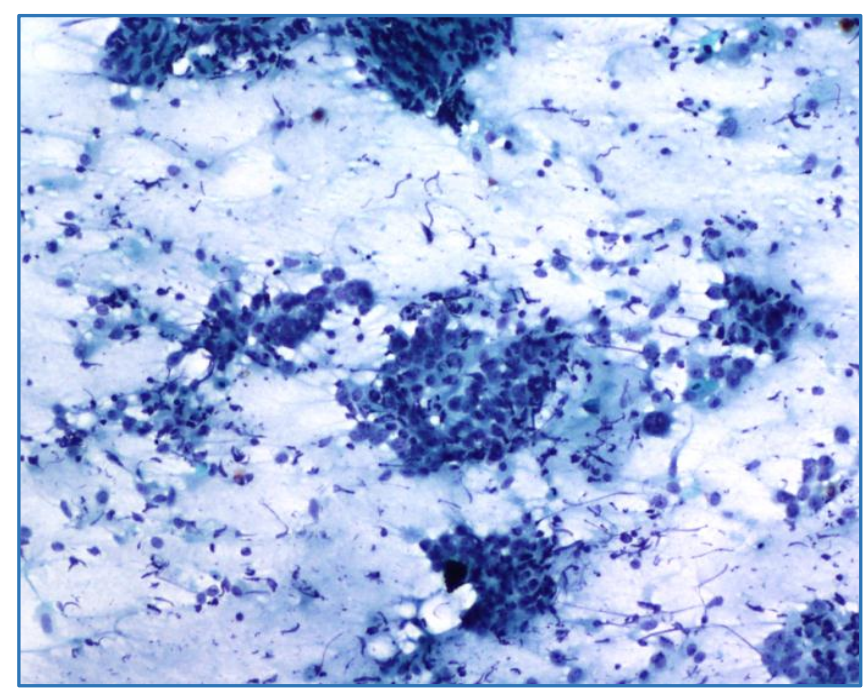

Fig. 7: Malignant Squamous Cells. Abundant Cytoplasm with Hyperchromatic, Pleomorphic Nuclei

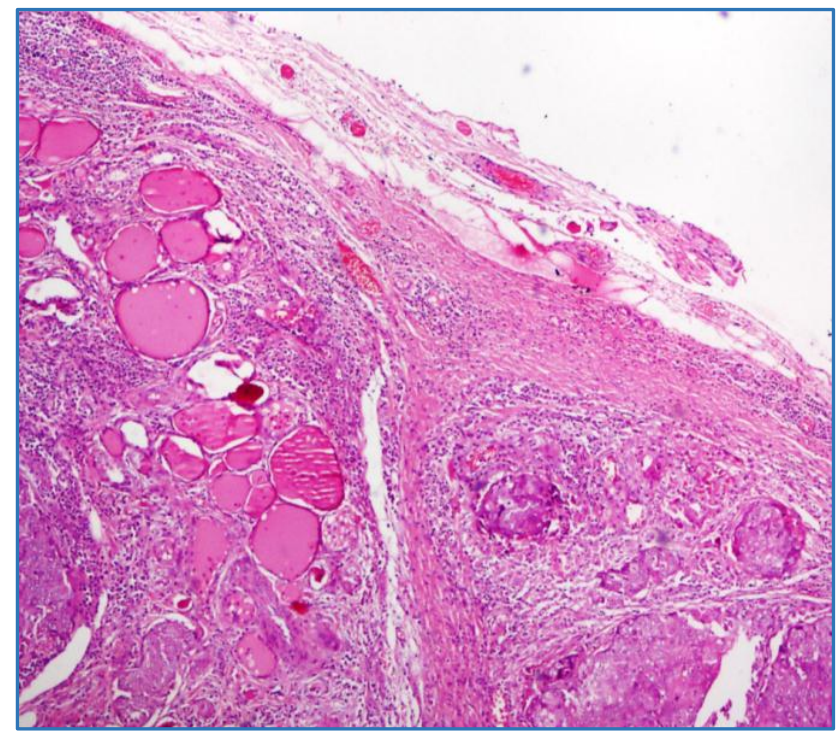

Fig. 8: Shows Thin Fibrous Capsule, Thyroid Follicles lined by Follicular Cells containing Colloid and Tumour composed of Malignant Squamous Cells in sheets

In view of the close margin on histopathology of the excised specimen and the presence of positive lymph nodes, external beam radiotherapy at a dose of 66 Greys over 33 fractions was administered with concurrent weekly chemotherapy with Cisplatin $70 \mathrm{mg}$ for 3 weeks. The patient was on regular followup for a year and shows no signs of recurrence, residual disease or metastasis.

\section{DISCUSSION}

During the first weeks of embryonic development, the thyroid gland descends from the foramen caecum at the base of the tongue to its normal position at a point below the thyroid cartilage. The developing thyroid remains connected to the tongue via an epithelial tract known as the thyroglossal duct. Normally, the thyroglossal duct tract later solidifies and subsequently obliterates entirely by the fifth to tenth week of gestation.[5] Thyroglossal duct cyst is the most common benign cervical mass except for benign cervical lymphadenopathy. The incidence of carcinoma in thyroglossal duct cyst is very rare, the incidence of which is $1 \%$. The majority of these are papillary carcinoma. The different histopathological types of neoplasia reported in cases of thyroglossal cyst malignancy are papillary carcinoma accounting for $81.7 \%$ of the cases, mixed papillary and follicular carcinoma accounting for $6.9 \%$ of the cases, squamous cell carcinoma accounting for $5.2 \%$ of the cases, follicular and adenocarcinoma accounting for $1.7 \%$ of the cases each and malignant stroma, epidermoid carcinoma and anaplastic carcinoma accounting for $0.9 \%$ of the cases each.[3]

The patient in this case report presented with a swelling in front of the neck with an FNAC report positive for squamous cell carcinoma. With such a cytological report, the following differential diagnoses were kept in consideration. Firstly, that it was a metastatic squamous cell tumour deposit from another primary site that is commonly affected such as the larynx and hypopharynx. However, on examination investigation in the form of CT scan and on panendoscopy there was no evidence of a primary growth at these sites. Biopsy of an area of congestion only revealed moderate dysplastic changes and the incidence of moderate dysplasia throwing metastasis is a rare entity. 
Further investigation in the form of ultrasonography of the abdomen was also performed to rule out the presence of other possible foci of primary tumour. The second possibility borne in mind was a primary squamous cell carcinoma of the thyroid gland itself. However, on examination and investigation in the form of CT scan of the neck, the thyroid gland was noted to be normal. Intraoperatively, the gland was also examined and found to be normal. A separate nodular lesion was noted, which was excised. The third possibility considered was a primary squamous cell carcinoma of a thyroglossal cyst. CT scan and intraoperative findings were in favour of the latter, but the diagnosis was clinched during histopathological evaluation of the excised specimen which revealed sheets of malignant squamous cells of moderate differentiation amongst thyroglossal duct cells.

A review of world literature reveals that only 25 cases of squamous cell carcinoma in a thyroglossal duct cyst reported till date with age ranging from 11 to 81 years. The patient in our case report was a male and fell near the same mean age (He was 49 years of age). Thyroglossal duct cyst malignancies usually present as a cystic midline neck mass that elevates on swallowing and protrusion of tongue. The patient in our case report presented similarly with a midline neck swelling, which moved with deglutition, but not on protrusion of the tongue because of its adherence to adjacent structures as it was harbouring a malignancy.

Among the various types of neoplasia in thyroglossal duct cyst, a papillary thyroglossal duct cyst carcinoma has the most favourable prognosis with occurrence of metastatic lesions in less than $2 \%$ of cases, while a squamous cell carcinoma has the worst prognosis.[6] The aetiology of squamous cell carcinoma, thyroid is uncertain as the thyroid gland lacks squamous epithelium. Three theories have been postulated in the development of squamous cell carcinoma. The first theory is the embryonic nest theory, which suggests that squamous cells are derived from embryonic remnants such as thyroglossal duct, thymic epithelium and ultimobranchial body.[7] The second theory is the metaplasia theory, which suggests that environmental stimuli (Inflammation and Hashimoto's thyroiditis) result in squamous metaplasia.[8] The third theory is the dedifferentiation theory which suggests that existing papillary, follicular, medullary and anaplastic thyroid carcinoma dedifferentiate into squamous cell carcinoma.[9]

Carcinomas arising from a thyroglossal cyst are usually characterized by non-aggressive behaviour and rare lymphatic spread.[10] Regional lymph node spread has occurred in only $7.7 \%$ of reported cases. ${ }^{[3]}$

The number of cases reported is too small for the formulation of a definitive and optimal treatment plan; however, surgery followed by radiotherapy seems to be the treatment of choice in most of the reported cases. Sistrunk's operation with or without lymph node dissection is done in most of the diagnosed cases. The role of postoperative radiation therapy has not been well defined in thyroglossal duct cyst carcinoma, partly because of the small number of reported cases; however, the general indications for radiotherapy include extension of the tumour beyond the cyst wall, microscopic extension of the tumour to the resected borders, locally advanced tumours (Invasion of tongue, larynx or thyroid), residual or recurrent disease and the presence of positive neck nodes.[11] Since the tumour in our case report had close margins and positive lymph nodes, the patient was subjected to postoperative concurrent chemoradiotherapy.

\section{CONCLUSION}

Thyroglossal duct cyst is the most common neck swelling usually located in the midline of the neck. The coexistence of carcinomas in thyroglossal duct cyst is extremely rare and hence its diagnosis may be missed. Diagnosis prior to excision is often difficult and definitive diagnosis can only be made postoperatively through histopathological evaluation of the excised specimen. Hence, importance is stressed on performing a Sistrunk's procedure or a total thyroidectomy with or without lymph node dissection for complete clearance of the tumour. Treatment with radioactive iodine or postoperative external beam radiotherapy has to be considered according to the clinical and microscopic findings. Accurate preoperative clinical and radiological evaluation is a must in order to identify this unique carcinoma.

\section{REFERENCES}

1. Shah S, Kadakia S, Khorsandi A, et al. Squamous cell carcinoma in a thyroglossal duct cyst: a case report with review of the literature. Am J Otolaryngol 2015;36(3):4602.

2. Peretz A, Leiberman E, Kapelushnik J, et al. Thyroglossal duct carcinoma in children: case presentation and review of the literature. Thyroid 2004;14(9):777-85.

3. Ellis PD, Van Nostrand AW. The applied anatomy of thyroglossal tract remnants. Laryngoscope 1997;87(5 Pt 1):765-70.

4. Bdesha AS, Layer GT. Primary papillary carcinoma of thyroglossal duct cyst report of a case and review literature. Br J Surg 1992;79(11):1248-9.

5. Maleki N, Alamdari MI, Feizi I, et al. Papillary carcinoma of the thyroglossal duct cyst: case report. Iran J Public Health 2014;43(4):529-31.

6. Martins AS, Melo GM, Tincani AJ, et al. Papillary carcinoma in a thyroglossal duct: case report. Sao Paulo Med J 1999;117(6):248-50.

7. Goldberg HM, Harrey P. Squamous cell cysts of the thyroid with special reference to the aetiology of squamous epithelium in the human thyroid. Br J Surg 1956;43:565-9.

8. Chaudhary RK, Barnes EL, Myers EN. Squamous cell carcinoma arising in Hashimoto's thyroiditis. Head Neck 1994;16(6):582-5.

9. Bronner MP, LiVolsi VA. Spindle cell squamous cell carcinoma of the thyroid: an unusual anaplastic tumour associated with tall cell papillary carcinoma. Mod Pathol 1991;4(5):637-43.

10. Kazemi M, Assadi M, Kazemi AA, et al. Primary papillary carcinoma of thyroglossal duct cyst. Hell J Nucl Med 2006;9(1):39-40.

11. Hama Y, Sugenoya A, Kobayashi S, et al. Squamous cell carcinoma arising from thyroglossal duct remnants: report of a case and results of immunohistochemical studies. Surg Today Jpn J Surg 1997;27(11):1077-81. 